\title{
Analysis Method of Traffic Congestion Degree Based on Spatio-Temporal Simulation
}

\author{
Shulin $\mathrm{He}$ \\ Department of Management \\ Liaoning police Academy \\ Dalian 116036, China
}

\begin{abstract}
The purpose of this research is to design and implement a road traffic congestion and traffic patterns simulation (TPS) model and integrate it with extension-information model (EIM). The problems of road traffic simulation and control are studied according to the method of extension information model, and from the spatio-temporal analysis point of view. The rules of the traffic simulation from existence to evolution are analyzed using theories. Based on this study, the concept of traffic system entropy is introduced, and resulted in the establishment of a fundamental frame work for the road traffic simulation system based on extension spatio-temporal information system. Moreover, a practicable methodology is presented.
\end{abstract}

Keywords- road traffic simulation; extension information model; degree of traffic congestion; traffic congestion entropy; traffic congestion control system.

\section{INTRODUCTION}

Traffic congestion has been a major problem on roads around the world for many years. In modern cities, traffic on major roads is abundant, and steps have to be taken to keep the traffic flowing at an acceptable speed. The volume of road traffic has increased rapidly in recent years. In the Dalian, total road traffic has almost doubled since 2001 (see Figure 2.1). Forecasts from the Department for Police show that the volume of road traffic will continue to increase at an alarming rate. These forecasts, which in the past have been conservative estimates, suggest that traffic levels will increase by approximately 50\% between the years 2010 and 2020. If this is the case, then the causes and effects of traffic congestion need to be understood now or it is could become much worse a problem in the near future.

The purpose of this research is to design and implement a road traffic patterns and traffic congestion degree simulation (TPS) model and integrate it with extension-information model (EIM). Under current computer software development conditions, it is rather difficult to implement TPS, or other spatial-temporal based complex simulation models. Different approaches have been explored to build spatial-temporal simulation models of traffic system. Because of this technical barrier, spatial-temporal simulation modelers have to spend more time on technical issues, which complicates the application of classics model of traffic system and other spatial simulation theories. This research developed a dynamic traffic pattern simulation model from a static road traffic model [1].
This research has chosen commercial property robbery simulation as an example for integrating an extension spatial-temporal simulation model with EIM. The simulation model applied EIM to the traffic congestion likelihood evaluation formula from routine traffic activity theory (RAT). The simulation process runs through much iteration, each generating some individual traffic congestion. The accumulation of individual congestion reveals traffic patterns in space and time.

Another reason for choosing traffic congestion pattern simulation is to expand SP's application to invisible spatial-temporal processes. Most SP applications have occurred in ecology, urban planning and environment studies. One of the SP core elements is state variable. State variables represent the status of cells, which are the focus of the modeling. Land use type and number of pollutant particle are examples of state variable. Compared with visible phenomena, it is more difficult to simulate spatial-temporal changes of invisible phenomena with SP modeling. To simulation traffic congestion pattern developing process from a micro-level, first we need to find out the invisible phenomenon which broadcast over space and time, and set it as state variable. The other variables can then be related to the state variable directly or indirectly.

This paper is organized as follows: Section 2 introduces some basic concepts about traffic congestion and system simulation. Section 3 presents our new simulation model of traffic congestion degree, and section 4 explains our proposal for traffic congestion control by self-organizing theory. Finally, section 5 concludes the paper and draws some future work.

\section{TRAFFIC CONGESTION AND SYSTEM SIMULATION}

\section{A. Traffic congestion}

Physicists have been trying to describe the phenomena of traffic for at least half a century. In the 1950s, James Lighthill, an expert on the physics of fluid flow, suggested that the flow of traffic on a road was akin to the flow of liquid in a pipe. This theory (the Lighthill-Whitham-Richards model) represented the flow of traffic entirely with mathematical equations, and ignored the individual drivers. This sort of model is called macroscopic, and can often produce realistic output, but lacks the complexity to model realistic driver behaviours [2]. 
The complexity and uncertainty of traffic congestion make an ideal illustration of such research. Traffic congestion patterns develop over time period as a result of interactions between target and congestion over space. At its most basic, congestion is caused when the volume of traffic exceeds road capacity. This holds for most perceived causes of congestion; for example, accidents, breakdowns and road works decrease the available road capacity, while school-run and holiday traffic increase the volume of traffic. The next approach was to treat vehicles as individual units instead of a continuous flow, and see what behaviour emerges when the vehicles are given simple rules to follow. Each vehicle would move according to the vehicle ahead, speeding up or slowing down to match its speed while maintaining a safe distance between cars. This is a type of microscopic model, which can vary in complexity depending on the aims of the simulation. One well-known model is a cellular automata model designed by Nagel and Schreckenberg [5]. It was very simplistic and followed mainly the rules above, yet exhibits complex phenomena found in real traffic, as described below.

The results from these models and from traffic studies show that flow rate and traffic density are linked in an interesting way. Normally, flow rate increases as density increases, that is, more vehicles are on the road without any having to slow down. However, when the density reaches a so-called 'critical density', the flow rate begins to decrease and the traffic becomes congested. An interesting observation is a hysteresis effect that as the density increases above the critical density it is possible for the flow to continue to increase in a met stable or bi-stable state. In this state, any hiccup in the flow can cause the traffic to become congested [3].

\section{B. System Simulation}

We can find from the whole procedure of traffic simulation, bring forward traffic system property's all kinds of believes basing on the information of traffic spatial-temporal. And then subjunctive prisoner and quality's possibility basing on a variety of suppose. Recurrent subjunctive procedure may form constringency. Constringency fixes traffic system information. This traffic system information is called for proof. Because of it, traffic system law can be obtaining. Obtain depend on themselves feeling and recognize abilities to get traffic system information from all the directions. If in this conditions, obtain works can be done; otherwise, it will be shrivel. In reality, the work that finding traffic system information from expressional information is very difficult, mainly reason is that we can't determine its resolution frame and information environment. Only depend on brain to finish quantity information development and constringe, to get to the purpose is very difficult.

To improve function and benefit of traffic system management and to build subjunctive traffic system model (TSM) is necessary. First definitude, TSM is tools dealing with traffic information in extension information space. TSM of extension information space is concurrence system that persons and computer are composed. The special complicacy and uncertainty of traffic system question are no having traffic's internal rules. For example, traffic congestion when happened, where, what form, why, what change? These are named for $5 \mathrm{CW}$ problem. It is over the barrel basing on mathematics in hand for the question. The mainly content of traffic information reality system consisted of $5 \mathrm{CW}$, its possibility space with mapping and inversion is consisting of element of crime simulation system.

In traffic system simulation, traditional ways are imitating inexact and uncertainty problem by building mathematics model, and then solve them by statistics and probability. But the model need a lot of believe and approximate, so at last, the model is different from reality largely. Obviously, traditional quantitative ways haven't satisfied with needs. For being short of information and unstructured, it isn't possible that building exact mathematical model; at the same time, decision aim of the question is miserable, so it is not necessary to building exact mathematics model. One practical way is building some qualitative models to analysis qualitatively. So make out some benefit analysis result, hand farthest knowledge's effect. Canon computer is information conduct system building on number arithmetic. Therefore, in traffic reality system researching, for deleting expression and model from computer, usually building definite believe and data's shortcut. In fact, those believes of subjunctive system aren't being at all. So we need an information digging technology what is fit for reality question.

Resolution for traffic management system expressed that traffic information is a simulation system of time and space. But information's unbending and asymmetric are formed by covert order of traffic properties causal order. Extension causal order searched relation order of causal order. And then it changed covert order into discoverable order in the traffic information, extended traffic expression information. We can get act information from expression information. Traffic system will reach to the purpose imitating and simulating under the causal mechanism. For example, in subjunctive traffic reality system, when computer gives us crime expressions, subjunctive system will appear causal relation images of traffic system properties. And then we can get traffic state expression information's relation information by alternant feeling procedure of person with computer.

\section{TRAFFIC SIMULATION BASED ON EXTENSION INFORMATION MODEL}

\section{A. Methodologies}

As an introduction to traffic simulation analysis, this section provides the definition of traffic simulation analysis as a general concept as well as definition of four types of traffic analysis. These definitions are meant to enhance the understanding of traffic system simulation analysis and to help create commonly understood terminology, concepts and ideas in the field of traffic system analysis.

The quantitative and qualitative studies of traffic system and law enforcement information in combination with social demographic and spatial factors to apprehend traffic, prevent congestion, reduce accident, and evaluate organizational procedure. From the definition, a number of data are required in analyzing traffic system so as to come up with informed decisions in the apprehension of traffic and planning. In order to understand the definition of system analysis, major phrases 
and terms used are defined and discussed in detail in the following $[5,6]$.

Traffic system simulation analysis uses both qualitative and quantitative data and it also uses analytical techniques. Qualitative data and analytical techniques refer to non-numerical data as well as the examination and interpretation of observations for the purpose of discovering underlying meanings and pattern of relationships. Quantitative data are data primarily in numerical or categorical format. Quantitative analysis consists of manipulations of observations for the purpose of describing and explaining the phenomena that those observations reflect and is primarily statistical.
Traffic system simulation analysis employs both types of data and techniques depending on the analysis and practical need. The information such as date, time, location, and type of traffic congestion is quantitative in that statistics can be used to analyze these variables. On the other hand, narrative of traffic congestion information are considered qualitative data in that a large number of narratives are nearly impossible to analyze statistically and are primarily examined to determine general themes and patterns. Three major elements of traffic simulation analysis emerge from the definition and these are traffic risk, spatial and temporal data as shown in table 1 below.

TABLE1. MAJOR FACTORS OF TRAFFIC SIMULATION

\begin{tabular}{|c|c|c|c|}
\hline & \multicolumn{3}{|c|}{ Factor } \\
\hline Phase & Human & Vehicle & Environment \\
\hline Pre-crash & $\begin{array}{l}\text { Information } \\
\text { Attitudes } \\
\text { Impairment } \\
\text { Police enforcement }\end{array}$ & $\begin{array}{l}\text { Roadworthiness } \\
\text { lighting } \\
\text { Braking } \\
\text { handling } \\
\text { Speed management }\end{array}$ & $\begin{array}{l}\text { Road design } \\
\text { Road layout } \\
\text { Speed limits } \\
\text { Pedestrian facilities }\end{array}$ \\
\hline Crash & $\begin{array}{l}\text { Use of restrains } \\
\text { Impairment }\end{array}$ & $\begin{array}{l}\text { Occupant restraints } \\
\text { Other safety devices } \\
\quad \text { Crash-protective design }\end{array}$ & Forgiving roadside \\
\hline Post-crash & $\begin{array}{l}\text { First-aid skill } \\
\text { Access to hospital }\end{array}$ & $\begin{array}{l}\text { Ease of access } \\
\text { Fire risk }\end{array}$ & $\begin{array}{l}\text { Rescue facilities } \\
\text { Congestion }\end{array}$ \\
\hline
\end{tabular}

Traffic simulation analysis is performed for different purposes and because of this, it has been sub divided into different categories which have been given specific names for the purpose. The following are four type of analysis that fall under the umbrella of traffic simulation analysis. Each contains characteristics of traffic simulation analysis in general, but each is specific in the type of data and simulation analysis used as well as in its purpose.

Traffic congestion is an extremely complicated social phenomenon, and has the features of random mutation according to its occurrence, development and the trend of its evolution. From the viewpoint of government management, society security and traffic accident prevention is the aim of traffic management. According to the existing concept on traffic system control, the control process is determined by three factors:

(1) Determine the possible space and time of traffic congestion;

(2) select some states from the possible space and time as targets;

(3) Create the necessary conditions to make the traffic system control reach the preset aim.

It is known that the term "possible space" is the assembly of all the possibilities faced in the development process of an object. The possible space of traffic congestion is determined by the conditions leading to a congestion case. These factors have their characteristic possible spaces and time, differing from each other in the amounts and the forms, and may interchange from one to another. When a possible space and time of a congestion case is developing into a certain state, it may turn into a new possible space and time.

The occurrence of several possible spaces and times in the development of traffic congestion makes the traffic process appearing in different stages. In other words, the target of traffic control changes as the possible congestion space varies.

\section{B. Simulation model of traffic congestion degree}

The point of quantitative traffic study lies in the traffic congestion situation relationship structure. It establishes situation model and relationship model. By combining them together, we get relationship structure analysis of practical traffic congestion.

The form is that we use the mathematical model coming from the quantitative analysis to get the key, then based on which we make qualitative inference [7,8]. At the same time, according to the relationship structure set mapping which gets from qualitative inference, we establish mathematical model through all kinds of set mapping space hypothesis. Two results will be fed back through self-organized relationship structure and finally form a feasible result. If road traffic system is made up of variables $C_{1}, C_{2}, \ldots, C_{n,}$, and there are controlling parameters $K_{1}, K_{2}, \ldots, K_{m}$, then traffic congestion dynamic equation is described as the following[6]: 


$$
\begin{aligned}
& \frac{d C_{1}}{d t}=f_{1}\left(\lambda, C_{1}, C_{2}, \Lambda, C_{n}\right) \\
& \frac{d C_{2}}{d t}=f_{2}\left(\lambda, C_{1}, C_{2}, \Lambda, C_{n}\right) \\
& \Lambda \Lambda \Lambda \Lambda \Lambda \Lambda \Lambda \Lambda \Lambda \Lambda \Lambda \Lambda \\
& \frac{d C_{n}}{d t}=f_{n}\left(\lambda, C_{1}, C_{2} \Lambda, C_{n}\right)
\end{aligned}
$$

In road traffic system, congestion situation variable is the key factor to form traffic system. The effective choice of situation variable is critical to reflect the real level of traffic management and describe social stability and the degree of the social development. The economic growth and consumption level reflect traffic system development. In economy study, if we lack the analysis of crime factors, our conclusion of the economy analysis will not be reliable. Among the former study of road traffic, there are many factors constructing traffic system, such as economy factor, education factor, social ethos, law factor, people relationship and management factor. Because these factors lie in different situations, the characteristics of traffic system are different. The evaluation of social stability and development depends on the choice of controlling parameter. For example unemployment rate, relative number of income difference, controlling proportion of informal social groups, management efficiency of road traffic and education quality.

$$
\frac{d C_{i}}{d t}=f_{i}\left(\left\{C_{j}\right\},\left\{k_{a}\right\}\right) \quad(i, j=1,2, \ldots, n a=1,2, \ldots, m)
$$

Suppose equation (2) is self-organization power traffic system, this system acts with a sudden change that has the statistical characteristics. But traffic action is invisible. The asymmetry of information makes it uncertain in the economic loss and investment when we solve cases and control traffic system. Therefore, the statistical prediction in traffic congestion degree and efficient estimate in solving traffic congestion cases are very important in macroeconomic analysis and growth, together with in the control the traffic stability.

Traffic congestion case is looked as the degree of congestion $n(t)$, which is decided by time $(t)$ 's function. It happens randomly. Because congestion happening and solving congestion cases are two variables, and the degree of congestion is decided by these variables, we suppose expectation of congestion degree $E\{n(t)\}=n(t)$, then

$$
E[n(t)]=N(t)=\sum_{n \rightarrow 0}^{\infty} n P_{n}(t)
$$

In the formula mentioned above, $P_{n}(t)$ indicates that time $(t)$ has $n$ congestion rate. Above all, by instinct, the changing rate of the expectation congestion degree, whose rate changes with time, is $d N(t) / d t$. During the time $(t)$, this happening rate explains the new congestion and repeated congestion. So in a certain area, the whole expected rate of congestion degree is that average rate $\lambda(t)$ counted on vehicle multiplies the expected congestion degree. If $\mu(t)$ is average rate of control degree of traffic congestion at time $t, \mu(t) n(t)$ is the general expected rate. So

$$
\frac{d N(t)}{d t}=[\lambda(t)-\mu(t)] N(t)
$$

This is called simulation model of traffic congestion degree. By analyzing the congestion attribute in certain area, if the traffic congestion doesn't transfer between inside and outside, i.e. specific rate of congestion degree and cracking rate doesn't change with time, then $\lambda(t)=\lambda, \mu(t)=\mu$, formula (3) will change into

$$
\frac{d N(t)}{d t}=(\lambda-\mu) N(t)
$$

In formula (4) result could be validated by replacing.

$$
N(t)=N(0) e^{(\lambda-\mu) t}
$$

In the formula, $N(0))$ is congestion degree when time is $\lambda-\mu=0$. When $\lambda-\mu>0$, it explains that practical congestion cracking is less than practical congestion degree. From study of recessive situation, we know that the number that people didn't report the situation and traffic congestion haven't been found is great many. So controlling aim of traffic system is $\lambda-\mu \rightarrow 0$. We find from above analysis that the indication of congestion degree has great effect on social development, especially on economy. If there is no traffic economy dynamic analysis in macroscopic traffic analysis, macroscopic traffic analysis will be half-baked. Here is congestion number interval [ $N_{\min }, N_{\max }$ ]; the degree of congestion situation happening is controlled among interval. Supposing two trends, $N(t) \rightarrow N_{\min }$ and $N(t) \rightarrow N_{\max }$, traffic congestion controlling degrees $k_{\alpha}$ and $k_{\beta}$ are replaced by $\lambda(t)-\mu(t)$ then Getting result of differential equation.

$$
\begin{aligned}
& k_{\alpha}=[\lambda(t)-\mu(t)]_{\alpha}=\left[1-N(t) / N_{\text {min }}\right](\lambda-\mu) \\
& k_{\beta}=[\lambda(t)-\mu(t)]_{\beta}=\left[1-N(t) / N_{\text {max }}\right](\lambda-\mu)
\end{aligned}
$$$$
[N(t)]_{\alpha}=\frac{N_{\min } e^{(\lambda-\mu) t}}{\left(N_{\min } / N(0)-1+e^{(\lambda-\mu) t}\right.},
$$

$$
[N(t)]_{\beta}=\frac{N_{\max } e^{(\lambda-\mu) t}}{\left(N_{\max } / N(0)-1+e^{(\lambda-\mu) t}\right.}
$$


Here supposing that the degree of traffic congestion controlling is chosen randomly between $k_{\alpha}$ and $k_{\beta}$ and. logarithm $\left[k_{\alpha}, k_{\beta}\right]$ is the ideal key to traffic congestion controlling. It can be inferred from the following. Formula (6) shows the general number characteristics of traffic congestion controlling level over a certain period of time [9].

$$
\begin{gathered}
{[N(t)]_{c}=\int_{t_{0}}^{t_{n}} \frac{[N(t)]_{\alpha}+[N(t)]_{\beta}}{2} d t} \\
=\int_{t_{0}}^{t_{n}}\left[\left[\frac{N_{\min } e^{(\lambda-\mu) t}}{\left(N_{\min } / N(0)\right)-1+e^{(\lambda-\mu) t}}+\right.\right. \\
\left.\left.\frac{N_{\max } e^{(\lambda-\mu) t}}{\left(N_{\max } / N(0)\right.}\right)-1+e^{(\lambda-\mu) t}\right] 2^{-1} d t
\end{gathered}
$$

Through the above discussion we know that the key point determining $[N(t)]$ is to get $\lambda(t)$ and $\mu(t)$, and the key point getting result is to establish equation (1) and (3). Establishing equation (1) is decided by analysis of congestion situation variable and equation (3)'s situation system structure. At the same time, congestion statistics and the methods of traffic information's measurement is very important.

\section{TRAFFic CONGESTION CONTROL}

\section{A. Non-equilibrium traffic control system}

In fact, the simulation system of traffic congestion is a self-organization system based on non-equilibrium system theory. Using non-equilibrium system theory to study the problems of traffic control is relatively new. Traffic congestion is a common social phenomenon. Traffic congestion prevention and control originate as congestion occur. The extent of the social stability is dependent on what level the traffic congestion is under control. Reducing the traffic congestion to the lowest extent means that the society advancing.

Philosophically, equilibrium exists temporarily and relatively. Non-equilibrium exists commonly and absolutely. The equilibrium achieved in the traffic congestion control system refers to the unified behavior of the control activity under the predictive traffic model. This is a static point of view for the traffic and their control. Equilibrium can be understood as a process of adapting with the traffic congestion and adjusting control, from the kinetic point of view. This reflects the characters of the non-equilibrium process. Since the traffic congestion appears to be kinetic in nature as the society is developing, traffic control system should be a non-equilibrium process.

However, the role of the traffic congestion information statistics should not be exaggerated. It has to be pointed out that the objectives processed by the statistics should be independent incidences and large numbers and have a random feature. Strictly speaking, the individual cases in a macroscopic traffic study do not fully fulfil the above mentioned assumptions. The statistics used before are not accurate and complete enough in describing the society traffic problem as a whole. Therefore, the needs are rising for a better theory to describe the social traffic thoroughly, and to take both microscopic and macroscopic viewpoints into consideration. From the view point of non-equilibrium system theory, the combination of statistics with kinetics should be useful in solving the problem mentioned before. The cooperation theory applies statistics for both microscopic and macroscopic investigations for the objectives under study.

The needs for a large number of objectives are no long a necessity. It is statistically meaningful on one hand, and it emphasizes the interactions in the social traffic on the other. Synergetic can be used to reveal the rules of the evolution of the traffic problems from the point of time, space and forms. Therefore, the social traffic problems can be described in a stricter and complete ways according to this theory, and the effective crime control can be found with the help of this comprehensive statistical method. The evolution of the traffic congestion problems is partly dependent on the changes of the traffic environment, and partly dependent on the control means and conditions of the government. According to the analysis of the mutation model, it is clear that the traffic congestion state model for studying the evolution pattern can be established by finding out the state variables representing the quantitative change, and the condition variables (i.e. controlling variables) which cause the change of the state of the crime-affected state in the social development.

For the analysis of the traffic congestion problem, the same principles apply. There are a number of factors, which affect the traffic congestion variations. The difficulty is to find out the dominant variables. All the traffic congestion variables can be divided into two groups: direct variables (directly affect the occurrence of traffic congestion). Once the relationship between the direct variables and the state variables is known, the mathematics model can be obtained, and the quantitative analysis can be carried out for the rules of the changes of the traffic state.

\section{B. The Entropy Characteristic of the traffic congestion system}

The traffic congestion system is non-equilibrium, so its feature is decided by the degree of the system disorder, which is determined by proper measurement. In physics, entropy denotes the amount of system probability, which quantitatively describes irreversible process. For traffic congestion system, any congestion situation couldn't happen again. It cannot restore. The macroscopic situation of traffic congestion system corresponds to many microcosmic traffic state. The more they correspond, the larger uncertainty of traffic system has. Thereby, it reflects the disorder of traffic system. It's obvious in traffic congestion problems. Because traffic action is complicated and changeable, and traffic action form and way are so various that we cannot predict. The characteristics in microcosmic traffic action situation can decide macroscopic congestion index of traffic system. Traffic system disorder can be measured by all kinds of congestion degree probability. Congestion entropy shows how much probability of each type of traffic action happens in traffic system. Supposing $\lambda_{c}$ is happening rate of congestion degree of macroscopic traffic 
system, and $k_{c}$ is traffic macroscopic controlling constant, then

$$
S^{c}=k_{c} \sum_{j=1}^{n} \ln \lambda_{c}^{j}
$$

$S^{C}$ is traffic congestion entropy, which is decided by adding trait and controlling constant of macroscopic traffic system $\lambda_{c}$.

In fact, traffic system is an open system. It shows that population flowing makes vehicle move between region inside and outside. Supposing the population in a region is $M$, in $M$ there are $M_{i c}$ people who probably commit a traffic action, then $d S_{i}=M_{i c} / M$ shows the change of inner entropy in traffic system. At the same time, due to the population flowing, the probability of traffic has changed, resulting in the change of exterior entropy. $d S_{o}=M_{o c} / M \quad M_{o c}$ is the number of flowing population in a region, and $M_{o c}$ is the number of traffic congestion probably happening among the flowing population. Then the change of entropy in traffic system is described as the following [9].

$$
\begin{aligned}
& d S^{c}=d S_{i}^{c}+d S_{o}^{c} \\
& d\left[k_{c} \sum_{j=1}^{n} \ln \lambda_{c}^{j}\right]=\frac{M_{i c}}{M}+\frac{M_{o c}}{M} \\
& \prod_{j=1}^{l} \lambda_{C}^{L}=e^{\frac{1}{k_{c}} \int_{T}\left(\frac{m_{i c}}{m}+\frac{m_{o c}}{m}\right)_{c} d t} \\
& (l \in(1,2, \Lambda, n))
\end{aligned}
$$

The general expected number of a regional traffic congestion doesn't always happen which must be combined with the rate of the congestion degree. We study the law of traffic system according to extension information changing model based on non-equilibrium system theory. At the same time, we will know how macroscopic traffic congestion control system affects social and economic system based on traffic congestion level and it's solving control index, together with changes of order parameter.

\section{CONCLUSIONS}

In this paper we have extended and improved a previous model for evaluating traffic congestion using an extension information model and applying a self-organizing methodology. We are improving function and benefit of traffic simulation and to build extension traffic simulation system (ETSS) is necessary.
First definitude, ETSS is tools dealing with information in extension information space. ETSS of extension information space is concurrence system that persons and computer are composed. The special complicacy and uncertainty of road traffic question are no having congestion's internal rules. For example, traffic congestion when happened, where, how, why, what change? These are named for $5 \mathrm{CW}$ problem. It is over the barrel basing on mathematics in hand for the question. The mainly content of road traffic information reality system consisted of $5 \mathrm{CW}$, its possibility space with mapping and inversion is consisting of element of road traffic simulation system.

In future research, by incorporating the information variables involved in traffic congestion management such as the numbers of accidents, traffic information violations, and traffic policemen on duty into an artificial intelligence technique, it is possible to build a traffic decision making system to help decision makers for analysis of traffic laws and policies.

\section{REFERENCES}

[1] Robert R, Theodore F. Contrasting the Use of Time-Based and Distance-Based Measures to Quantify Traffic Congestion Levels: An Analysis of New Jersey Counties. The 81th Annual Meetings of the Transportation Research Board, Washington, D.C, 2002.

[2] He Ping. Research on the Quantity Analysis of Social Crime. Journal of Liaoning Police Academy, 2004, vol. 37, pp.1-6.

[3] Kent Hymely. "Does Traffic Congestion Reduce Employment Growth?" Journal of Urban Economics, 2009,vol. 65/2, pp. 127-135.

[4] Kleijnen, J. P. C. Response surface methodology for constrained simulation optimization: An overview, Simulation Modelling Practice and Theory, 2008, vol.16, pp.50-64.

[5] Kleijnen, J. P. C., van Beers, W. and van Nieuwenhuyse, I. Constrained optimization in expensive simulation: Novel approach, European Journal of Operational Research, 2010, vol.202, pp.164-174.

[6] Marti, K. Stochastic optimization methods, Springer, Berlin. Mor'e, J. and Wild, S. (2009). Benchmarking derivative-free optimization algorithms, SIAM Journal on Optimization, 2009, vol.20, pp.172-191.

[7] Oeuvray, R. and Bierlaire, M. Boosters: a derivative-free algorithm based on radial basis functions, International Journal of Modelling and Simulation, 2009, vol. 29,pp.26-36.

[8] Osorio, C. Mitigating network congestion: analytical models, optimization methods and their applications, $\mathrm{PhD}$ thesis, Ecole Polytechnique F'ed'erale de Lausanne. Osorio, C. and Bierlaire, M. (2009a). An analytic finite capacity queueing network model capturing thepropagation of congestion and blocking, European Journal Of Operational Research, 2010, vol. 196, pp.996-1007.

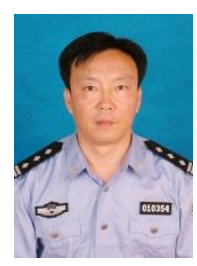

\section{AUTHORS PROFILE}

Shulin He was born in 1962, in Liaoyang, China. He is an assistant professor of the management department of the Liaoning Police Academy, Dalian, China. His academic interests are found in traffic management theory and applications, including uncertain traffic system analysis. $\mathrm{He}$ can be reached at e-mail: hesh1888597@yahoo.com.cn. 